\title{
Avaliação Prospectiva do Perfil Lipídico na Artrite Reumatóide
}

\section{Prospective Evaluation of the Lipid Profile in Rheumatoid Arthritis}

\author{
Alessandra Barbosa Avelar ${ }^{(1)}$, Ana Karla Guedes de Melo(1), Branca Dias Batista de Souza ${ }^{(2)}$
}

\section{RESUMO}

Objetivo: Avaliar a alteração do perfil lipídico na artrite reumatóide e sua associação com a atividade de doença. Métodos: Estudo prospectivo de 21 pacientes, do sexo feminino, com artrite reumatóide, acompanhados durante um ano. Foram avaliados colesterol total, LDL-colesterol, HDL-colesterol, triglicérides, apolipoproteínas A e B, lipoproteína (a) e VHS, além de HAQ e índice de atividade de doença, por meio do DAS28. Resultados: Após um ano, houve aumento significativo dos níveis de colesterol total $(\mathrm{p}=0,019)$ em $66,6 \%$ das pacientes e de LDL-colesterol $(\mathrm{p}=0,008)$ em $80,9 \%$ das pacientes. Quanto aos níveis de lipoproteína (a), houve redução significativa $(\mathrm{p}=0,003)$ em $33,33 \%$ dos casos. Diminuição significativa ocorreu também no VHS, HAQ e DAS28. Porém, não houve associação entre a alteração do perfil lipídico e a atividade de doença. Conclusão: Embora tenha sido significativa a redução da atividade da doença (DAS28), houve piora do perfil lipídico.

Palavras-chave: artrite reumatóide, lipoproteína, lípides, aterosclerose, inflamação.

\section{INTRODUÇÃO}

A artrite reumatóide (AR) é uma doença inflamatória crônica que afeta primariamente a membrana sinovial, levando ao dano articular e à destruição óssea ${ }^{(1)}$. Acomete $1 \%$ da população e está associada ao aumento de morbimortalidade por causa de doenças cardiovasculares (DCV), predominantemente a aterosclerose acelerada $a^{(2,3)}$.

Alterações no perfil lipídico, como baixos níveis de HDL ( high density lipoprotein)-colesterol, geralmente associados a altos níveis de colesterol total (CT), LDL (low density lipoprotein)-colesterol e triglicérides (TG), são responsáveis pela maior prevalência de $\mathrm{DCV}$, tanto na população geral quanto nos pacientes com $\mathrm{AR}^{(4)}$. Há evidências de que o

\begin{abstract}
Objective: To evaluate the lipid profile in rheumatoid arthritis and its association with the disease activity. Methods: This was a prospective study with 21 female patients who had rheumatoid arthritis and whose progress was accompanied for 1 year. Total cholesterol, LDL-cholesterol, HDL-cholesterol, triglycerides, apolipoproteins $A$ and $B$, lipoprotein (a), ESR, as well as $H A Q$ and disease activity through DAS28, were evaluated. Results: After 1 year, the total cholesterol level was increased in $66.6 \%$ of the patients $(p=0.019)$ and the LDL-cholesterol level was also elevated in $80.9 \%$ of the patients $(p=0.008)$. In contrast, the lipoprotein (a) level was reduced in $33.33 \%$ of the cases $(p=0.003)$. A significant decrease also occurred in ESR, HAQ and DAS28. However, there was no association between the alteration of the lipid profile and the disease activity. Conclusion: Although the reduction in the disease activity (DAS28) was significant, the lipid profile worsened.
\end{abstract}

Keywords: rheumatoid arthritis, lipoprotein, lipids, atherosclerosis, inflammation.

aumento de apolipoproteína B (apo B) está relacionado a maior risco cardiovascular, enquanto a apolipoproteína $\mathrm{Al}$ (apo Al) aumentada seria fator protetor para desenvolvimento de $\mathrm{DCV}^{(5,6)}$.

Estudos prévios sugeriram que fatores de risco cardiovasculares "tradicionais” não são os únicos responsáveis pelo aumento de complicações vasculares na AR, já que aproximadamente $50 \%$ da doença arterial coronariana aterosclerótica nestes pacientes ocorrem na ausência destes fatores ${ }^{(7,8)}$. Evidências sugerem que a inflamação é responsável pelo início e pela progressão da aterosclerose, tornando-se, assim, fator importante na AR e contribuindo para o desenvolvimento de aterosclerose $\operatorname{precoce}^{(9,10)}$.

\footnotetext{
Recebido em 12/11/2007. Aprovado, após revisão, em 5/6/2008. Declaramos a inexistência de conflitos de interesse.

Serviço de Reumatologia do Departamento de Medicina da Santa Casa de São Paulo.

1. Médica-Especialista em Reumatologia e Pós-Graduanda da Faculdade de Ciências Médicas da Santa Casa de São Paulo.

2. Professora da disciplina de Reumatologia da Faculdade de Ciências Médicas da Santa Casa de São Paulo (FCMSCSP) e chefe do Serviço de Reumatologia da Irmandade Santa Casa de Misericórdia de São Paulo.

Endereço para correspondência: Alessandra Barbosa Avelar. Santa Casa de Misericórdia de São Paulo, Hospital Central, Departamento de Medicina, Serviço de Reumatologia, Rua Dr. Cesário Mota Júnior, 112 - Vila Buarque, 01221-020, São Paulo, SP, e-mail: alebavelar@bol.com.br
} 
Pela associação dos fatores de risco tradicionais e nãotradicionais, o perfil lipídico na AR é pró-aterogênico e é exemplificado por avaliações que demonstraram baixos níveis de HDL-colesterol e aumento de lipoproteína (a) em pacientes com AR ativa ${ }^{(11-14)}$. A AR com manifestações extra-articulares está associada ao aumento da mortalidade cardiovascular em virtude da inflamação acentuada, exemplificando o papel da inflamação intrínseca da AR no dano vascular ${ }^{(15)}$.

Fundamentando-se nos dados da literatura, o presente estudo tem como objetivo avaliar a evolução do perfil lipídico e sua associação com a atividade de doença em pacientes com AR.

\section{PACIENTES E MÉTODOS}

Estudo prospectivo com duração de um ano (2006 a 2007), no qual foi avaliado inicialmente 30 pacientes do ambulatório de AR do Serviço de Reumatologia da Santa Casa de São Paulo, porém completaram o estudo 21 pacientes do sexo feminino. Todas as pacientes preenchiam os critérios do American College of Rheumatology para AR e tinham doença em atividade, definida pelo DAS28 (Disease Activity Index 28-Joint Score) maior que 2,6. Foram excluídas do estudo pacientes que estavam em uso de antimaláricos e uso de medicamentos hipolipemiantes.

As medicações utilizadas durante o estudo foram prednisona na dose média de $7,5 \mathrm{mg} /$ dia, metotrexate na dose média de $15 \mathrm{mg} / \mathrm{semana}$ (via oral), sulfassalazina $2 \mathrm{~g} /$ dia, leflunomide $20 \mathrm{mg} /$ dia e, em alguns casos, o uso de anti-TNF $(3 \mathrm{mg} / \mathrm{kg} /$ dose $)$. No início, introduzia-se a prednisona e uma droga modificadora do curso da doença (DMCD), caso o DAS28 se mantivesse elevado, outras DMCD eram associadas até atingirem remissão da doença (DAS28 < 2,6). Em casos refratários à associação de DMCD, optou-se pelo anti-TNF, conforme preconizado pelo Consenso Brasileiro no Diagnóstico e Tratamento da Artrite Reumatóide ${ }^{(16)}$. Foi utilizado o captopril na dose média de $70 \mathrm{mg} /$ dia, para as pacientes hipertensas (pressão arterial acima de $140 \times 90 \mathrm{mmHg}$ ). Todos os pacientes que usavam metotrexate fizeram uso de ácido fólico na dose média de 7,5 mg/semana.

Foram realizadas duas avaliações clínicas e laboratoriais, sendo uma no início do estudo e outra após um ano. Foi avaliada a atividade de doença por meio do DAS28 e a capacidade funcional pelo HAQ (bealth assessment questionnaire). Laboratorialmente, avaliou-se colesterol total, HDL-colesterol, LDL-colesterol, triglicérides, apo- lipoproteína A (VR 125-215 mg/dL), apolipoproteína B (VR 55-125 mg/dL), lipoproteína (a) $(\mathrm{VR}<54 \mathrm{mg} / \mathrm{dL}$ ) e velocidade de hemossedimentação (VHS $<12 \mathrm{~mm}$ ), portanto o método utilizado para dosagem de apolipoproteínas foi o imunonefelométrico e para a lipoproteína (a) foi o imunofotométrico. Estas dosagens foram realizadas pelo Laboratório Central e seu laboratório de apoio.

\section{ANÁLISE ESTATÍSTICA}

Os valores quantitativos foram demonstrados como média \pm desvio-padrão e foram comparados por meio do teste t de Student, considerando-se como estatisticamente significativo $o$ valor de $p<0,05$. Para verificar a associação entre o perfil lipídico e a atividade de doença foi utilizada a regressão linear, considerando DAS28 $<2,6$ como remissão da doença.

\section{RESULTADOS}

As características demográficas do estudo consistiram de 21 pacientes do sexo feminino, com idade média de $53,7 \pm$ 9,29 anos, com tempo médio de duração de doença de 6,83 $\pm 5,26$ anos, DAS 28 de $6,33 \pm 0,92$ e HAQ de $1,35 \pm 0,62$. Todas as pacientes apresentavam fator reumatóide positivo e tinham erosões à radiografia de mãos e/ou pés.

As pacientes apresentavam média de índice de massa corpórea (IMC) de $26,39 \pm 3,80 \mathrm{~kg} / \mathrm{m}^{2}$ e glicemia de $90 \pm 8,99 \mathrm{mg} / \mathrm{dL}$. Entre as 21 pacientes, duas $(9,5 \%)$ relataram história familiar positiva para doença coronariana, cinco $(23,8 \%)$ eram hipertensas e nenhuma diabética (Tabela 1).

Tabela 1

Características CLÍNicas E Laboratoriais das PACIENTES COM ARTRITE REUMATÓIDE $(\mathrm{N}=21)$

\begin{tabular}{lc}
\hline Idade (anos) & $53,7(9,29)$ \\
Mulheres & $100 \%$ \\
Tempo de doença (anos) & $6,83(5,26)$ \\
DAS28 & $6,33(0,92)$ \\
HAQ & $1,35(0,62)$ \\
IMC $\left(\mathrm{kg} / \mathrm{m}^{2}\right)$ & $26,39(3,80)$ \\
Glicemia $(\mathrm{mg} / \mathrm{dL})$ & $90(8,99)$ \\
Hipertensão arterial & $23,8 \%$ \\
História familiar de DCV & $9,5 \%$ \\
\hline
\end{tabular}

Disease Activity Index 28-Joint Scorehealth assessment questionnaire 
Quanto às medicações utilizadas, 21 (100\%) das pacientes usaram metotrexate, ácido fólico e prednisona, uma $(4,76 \%)$ paciente usou sulfassalazina, enquanto duas $(9,5 \%)$ utilizaram leflunomida e anti-TNF. As cinco $(23,8 \%)$ pacientes hipertensas fizeram uso de captopril (Tabela 2). Os antimaláricos não foram utilizados.

TABela 2

MedicaCõés, porcentagem de uso e dose média UTILIZADAS PELAS 21 PACIENTES COM ARTRITE REUMATÓIDE DURANTE O ESTUDO

\begin{tabular}{lcc}
\hline Medicação & Percentagem de uso (\%) & Dose média \\
\hline Metotrexate & 100 & $15 \mathrm{mg} / \mathrm{sem}$ \\
Prednisona & 100 & $7,5 \mathrm{mg} /$ dia \\
Sulfassalazina & 4,76 & $2 \mathrm{~g} / \mathrm{dia}$ \\
Leflunomida & 9,5 & $20 \mathrm{mg} / \mathrm{dia}$ \\
Anti-TNF & 9,5 & $3 \mathrm{mg} / \mathrm{kg} /$ dose \\
Ácido fólico & 100 & $7,5 \mathrm{mg} / \mathrm{semana}$ \\
Captopril & 23,8 & $70 \mathrm{mg} /$ dia \\
\hline
\end{tabular}

No início do estudo, as pacientes já apresentavam média de CT e LDL-colesterol acima dos níveis preconizados para a população de risco, além de possuírem alta atividade de AR, confirmada pela média do DAS28 maior que 5,1.

Após um ano houve alteração do perfil lipídico, bem como de VHS, DAS28 e HAQ. Quanto a modificação do perfil lipídico, ocorreu aumento significativo dos níveis de CT $(190,95 \mathrm{mg} / \mathrm{dL} \times 211,43 \mathrm{mg} / \mathrm{dL}, \mathrm{p}=0,019)$, observado em $14(66,6 \%)$ pacientes, e também dos níveis de LDL-colesterol $(113,86 \mathrm{mg} / \mathrm{dL} \times 129,81 \mathrm{mg} / \mathrm{dL}$, $\mathrm{p}=0,008)$, o que ocorreu em $17(80,9 \%)$ pacientes. Já os níveis de lipoproteína (a) sofreram redução significativa $(52,22 \mathrm{mg} / \mathrm{dL} \times 35,56 \mathrm{mg} / \mathrm{dL}, \mathrm{p}=0,003)$, ocorrendo em sete $(33,33 \%)$ pacientes. Não houve alterações quanto aos níveis de apolipoproteínas A e B, HDL-colesterol e triglicérides. Vale ressaltar que não foi detectada alteração do hábito alimentar e nem alteração do IMC ao final do estudo.

Redução significativa foi percebida na VHS, no DAS28 e no HAQ quando comparados após um ano. A VHS média era de $49,76 \mathrm{~mm}$ e foi para $20,86 \mathrm{~mm} \operatorname{com~} \mathrm{p}=0,02$. O HAQ inicial de $1,35 \pm 0,62$ comparado com $0,63 \pm 0,43$ no final do estudo $(\mathrm{p}=0,000)$. E, por último, a diminuição significativa do DAS28 que inicialmente era em média 6,33 e passou para $2,95(\mathrm{p}=0,000)$, portanto das 21 pacientes, oito $(38,09 \%)$ atingiram remissão da doença, justificada pelo DAS28 abaixo de 2,6 (Tabela 3).
Tabela 3

Comparação do Perfil líídico, DAS, HAQ E VHS NO INÍCIO E APÓS UM ANO NAS PACIENTES COM $\operatorname{AR}(\mathrm{N}=21)$

\begin{tabular}{lccc}
\hline Variável & Início & 1 ano & $\mathbf{p}$ \\
\hline Colesterol total $(\mathrm{mg} / \mathrm{dL})$ & $190,95(50,95)$ & $211,43(37,01)$ & 0,019 \\
HDL-colesterol $(\mathrm{mg} / \mathrm{dL})$ & $54,52(15,27)$ & $57,24(12,51)$ & 0,473 \\
LDL-colesterol $(\mathrm{mg} / \mathrm{dL})$ & $113,86(40,34)$ & $129,81(34,14)$ & 0,008 \\
Triglicérides $(\mathrm{mg} / \mathrm{dL})$ & $112,24(48,55)$ & $132,76(53,03)$ & 0,082 \\
Lipoproteína $(\mathrm{a})(\mathrm{mg} / \mathrm{dL})$ & $52,22(47,82)$ & $35,56(39,39)$ & 0,003 \\
Apolipoproteína A (mg/dL) & $157,88(28,08)$ & $157,47(26,65)$ & 0,964 \\
Apolipoproteína B (mg/dL) & $102,94(35,53)$ & $110(32,13)$ & 0,222 \\
DAS28 & $6,33(0,92)$ & $2,95(1,02)$ & 0,000 \\
HAQ (1-3) & $1,35(0,62)$ & $0,63(0,43)$ & 0,000 \\
VHS (mm) & $49,76(29,98)$ & $20,86(20,17)$ & 0,02 \\
\hline
\end{tabular}

Dados em média (desvio-padrão); DAS28 = Disease Activity Index 28-Joint Score; $\mathrm{HAQ}=$ health assessment questionnaire $(1-3) ; \mathrm{VHC}=$ velocidade de hemossedimentação.

O presente estudo não encontrou associação entre alteração do perfil lipídico e atividade de doença, avaliada pelo DAS28.

\section{DISCUSSÃO}

Observa-se no presente estudo aumento significativo dos níveis de CT e LDL-colesterol e redução significativa da lipoproteína (a) após um ano, embora não se tenha correlacionado à atividade de doença, avaliada pelo DAS28. Quanto aos níveis de apolipoproteínas A e B, triglicérides e HDL-colesterol não houve diferença estatística. Os dados da literatura quanto à relação entre perfil lipídico e AR são bastante controversos. Há estudo mostrando que não há mudança no perfil lipídico após 48 semanas de tratamento com anti-TNF ${ }^{(17)}$, enquanto outro demonstra aumento significativo dos níveis de HDL-colesterol após tratamento, porém não se correlacionando com o DAS28, isto é, atividade de doença ${ }^{(18)}$. Acredita-se que a inflamação exerça papel central no desenvolvimento da aterosclerose, pois a inflamação crônica promove o aumento dos biomarcadores, com ativação de células endoteliais e disfunção vascular, levando à diminuição da complacência do vaso e ao surgimento da placa de ateroma, entretanto, a razão do aumento de DCV na AR é complexa e não totalmente esclarecida ${ }^{(19)}$. Evidências sugerem que a inflamação da AR esteja associada a perfil lipídico desfavorável e, dessa forma, seria fator de risco independente para doenças cardíacas 
isquêmicas precoces ${ }^{(20-22)}$. Tal fato pode ser exemplificado por um estudo que demonstra que a inflamação contribui para o perfil lipídico mais aterogênico em pacientes com $A R$, dez anos antes de desenvolverem a clínica da doença ${ }^{(23)}$. Atribui-se o aumento dos níveis de CT e LDL-colesterol no início do presente estudo ao tempo médio de doença das pacientes (6,83 anos), e, em virtude do pequeno número de pacientes e a pequena porcentagem do uso de outras medicações, que não o metotrexate e a prednisona, não se pode afirmar que a piora do perfil lipídico se deva ao uso de medicação. Apesar de se conseguir diminuição significativa de HAQ, VHS e DAS28, apenas 38,09\% das pacientes atingiram remissão da doença, ou seja, DAS28 < 2,6. Na literatura há estudos controversos quanto à lipoproteína (a) e às apolipoproteínas A e B na AR, pois alguns estudos mostram a associação entre lipoproteínas (a) e proteínas de fase aguda, principalmente a proteína $\mathrm{C}$ reativa, enquanto outros não suportam este fato ${ }^{(24)}$. No presente estudo demonstrou-se redução significativa da lipoproteína (a), embora não se tenha correlacionado com a atividade de doença (DAS28). Uma possível explicação para esta diminuição seria a redução considerável do VHS após um ano, pois, como a lipoproteína (a) é proteína de fase aguda, com a diminuição do VHS, pode-se inferir que houve melhora

\section{REFERÊNCIAS}

1. Gravallese EM: Bone destruction in arthritis. Ann Rheum Dis 61(Suppl 2): 84-6, 2002.

2. Van Doornum S, McColl G, Wicks IP: Accelerated atherosclerosis: an extraarticular feature of rheumatoid arthritis?. Arthritis Rheum 46: 862-73, 2002.

3. Riise T, Jacobsen BK, Gran JT, et al.: Total mortality is increased in rheumatoid arthritis. A 17 -year prospective study. Clin Rheumatol 20: 123-7, 2001.

4. Castelli Wp, Garrison RJ, Wilson PW, et al.: Incidence of coronary heart disease and lipoprotein cholesterol levels. The Framingham Study. JAMA 256: 2835-8, 1986.

5. Walldius G, Jungner I: Apolipoprotein B and apolipoprotein A-1: risk indicators of coronary heart disease and targets for lipid modifying therapy. J Intern Med 255: 188-205, 2004.

6. Luc G, Bond J, Evans A, et al.: Value of HDL cholesterol, apolipoprotein A-I, lipoprotein A-I, and lipoprotein A-I/A-II in prediction of coronary heart disease, the PRIME study. Arterioscler Thromb Vasc Biol 22: 1155-61, 2002.

7. del Rincon ID, Willians K, Stern M, et al.: High incidence of cardiovascular events in a rheumatoid arthritis cohort not explained by traditional cardiac risk factors. Arthritis Rheum 44: 2737-45, 2001.

8. Ross R: The pathogenesis of atherosclerosis: a perspective for the 1990s. Nature 362: 801-9, 1993. do processo inflamatório, embora não se tenha avaliado a proteína $\mathrm{C}$ reativa, que é o que mais se relaciona à lipoproteína (a) como sugerido em estudos prévios. Enquanto se observa na literatura vários resultados quanto aos níveis de apolipoproteínas, não se conseguiu mostrar alteração significativa. Este estudo diferiu de outros, porque não foi obtida melhora considerável nos níveis de HDL-colesterol e nem diminuição de CT e LDL-colesterol após o tratamento, talvez pelo fato de a população em estudo ter maior tempo médio de doença e ser composta apenas pelo sexo feminino, quando comparada a de outros estudos, que, geralmente, são pacientes com AR de início recente (aproximadamente um ano de doença), e de ambos os sexos.

Concluindo, o presente estudo demonstrou piora do perfil lipídico das pacientes com AR após avaliação de um ano, independente da atividade de doença. Pois, não obstante a significativa melhora da atividade da doença demonstrada pela redução importante do DAS28, as pacientes evoluíram com piora do perfil lipídico e, dessa forma, possuem maior risco cardiovascular. Portanto, ressalta-se a importância de se tratar igualmente os fatores de risco tradicionais e não-tradicionais, por meio do tratamento da própria doença, a fim de reduzir a morbimortalidade cardiovascular da AR.

9. Bergholm R, Leirisalo-Repo M, Vehkavaara S, et al.: Impaired responsiveness to NO in newly diagnosed patients with rheumatoid arthritis. Arterioscler Thromb Vasc Biol 22: 1637-41, 2002.

10. Danesh J, Whincup P, Walker M, et al.: Low grade inflammation and coronary heart disease: prospective study and update metaanalyses. BMJ 321: 199-204, 2000.

11. Park YB, Lee SK, Lee WK, et al.: Lipid profiles in untreated patients with rheumatoid arthritis. J Rheumatol 26: 1701-4, 1999.

12. Choi HK, Seeger JD: Lipid profiles among US elderly with untreated rheumatoid arthritis-the Third National and Nutrition Examination Survey. J Rheumatol 32: 2311-6, 2005.

13. Yog WH: Dyslipoproteinemia in patients with active rheumatoid arthritis: effects of disease activity, sex and menopausal status on lipid profiles. J Rheumatol 31: 1746-53, 2004.

14. McMahon M, Grossman J, Fitzgerald J, et al.: Pro-inflammatory HDL as a biomarker for atherosclerosis in SLE and RA. Arthritis Rheum 52: S697-8, 2005.

15. Vis M, Nurmohamed MT, Wolbink G, et al.: Short term effects of infliximab on the lipid profile in patients with rheumatoid arthritis. J Rheumatol 32: 252-5, 2005.

16. Bértolo $\mathrm{MB}$, Brenol $\mathrm{CV}$, Schainberg $\mathrm{CG}$, et al.: Atualização do consenso brasileiro no diagnóstico e tratamento da artrite reumatóide. Rev Bras Reumatol 47: 151-9, 2007. 
17. Peters MJL, Vis M, van Halm VP, et al.: Changes in lipid profile during infliximab and corticosteroid treatment in rheumatoid arthritis. Ann Rheum Dis 66:958-61, 2007.

18. Georgiadis AN, Papavasiliou EC, Lourida ES, et al.: Atherogenic lipid profile is a feature characteristic of patients with early rheumatoid arthritis: effect of early treatment - a prospective, controlled study Arthritis Res Ther8:R82, 2006.

19. Schillinger $M$, Exner $M$, Miekusch W, et al.: Inflammation and Carotid Artery-Risk for Atherosclerosis Study (ICARAS). Circulation 111: 2203-9, 2005.

20. Lee YH, Choi SJ, Ji JD, et al.: Lipoprotein (a) and lipids in relation to inflammation in rheumatoid arthritis. Clin Rheumatol 19: $324-5,2000$.
21. Dursunoglu D, Evrangul H, Palat B, et al.: Lp(a) lipoprotein and lipids in patients with rheumatoid arthritis: serum levels and relationship to inflammation. Rheumatol Int 25: 241-5, 2005.

22. Bacon PA, Stevens RJ, Carruthers DM, et al.: Accelerated atherogenesis in autoimmune rheumatic diseases. Autoimmun Rev 1: 338-47, 2002.

23. van Halm VP, Nielen MMJ, Nurmohamed MT, et al.: Lipids and inflammation: serial measurements of the lipid profile of blood donors who later developed rheumatoid arthritis. Ann Rheum Dis 66: 184-8, 2007.

24. Rantapaa-Dahlqvist S, Wallberg-Jonsson S, Dahlen G: Lipoprotein (a), lipids, and lipoproteins in patients with rheumatoid arthritis. Ann Rheum Dis 50: 366-8, 1991. 